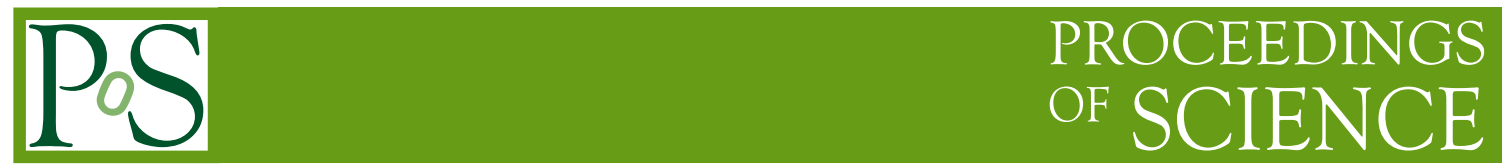

\title{
Recent heavy ion results from CMS
}

\author{
Subash Chandra Behera for the CMS Collaboration* \\ Indian Institute of Technology, \\ Madras, Chennai, India \\ E-mail: subash.chandra.behera@cern.ch
}

The conditions in collisions of ultra-relativistic nuclei correspond to a very early time of the universe. At CERN's Large Hadron Collider (LHC), studies of the properties of the produced hot and dense strongly-interacting matter are carried out by all four big experiments. In this talk, we will give an overview of recent exciting heavy-ion results from the Compact Muon Solenoid experiment. We will cover different areas of interest, such as, heavy-flavour probes on Quark Gluon Plasma (QGP), initial states, and bulk QGP physics.

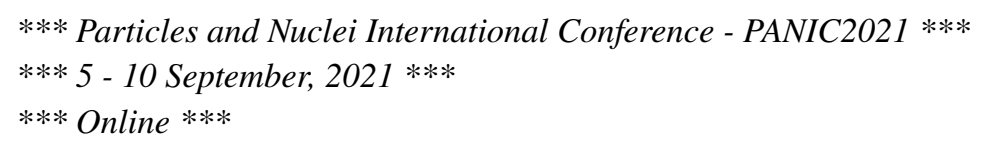

${ }^{*}$ Speaker 


\section{Introduction}

One of the major challenges of heavy-ion physics is to understand the properties of the hot and dense medium, known as Quark Gluon Plasma (QGP), produced in ultra-relativistic nucleusnucleus collisions [1]. Various probes, such as heavy-flavour hadrons and electroweak bosons, are used to understand more about the properties of heavy-ion collisions such as the initial state geometry, including the spatial distribution of nucleons and their fluctuations. In addition, other observables are used to study the QGP medium effects, such as particle correlation, jet-quenching, flow etc. [2]. This note presents the overview of various recent interesting and exciting heavy-ion results from the CMS experiment [3].

\section{Initial state interaction}

In this section, we will discuss the $T_{A A}$-normalized yields of $\mathrm{Z}$ bosons calculated as a function of centrality in lead-lead $(\mathrm{PbPb})$ collisions. We will also discuss the $\Delta v_{2}$ from prompt $D^{0}$ and $\overline{D^{0}}$. Finally, we will cover some exciting results in multiplicity dependence of dimuon acoplanarity and mean mass.

The $\mathrm{Z}$ boson azimuthal anisotropy $\left(v_{2}\right)$ as a function of centrality is shown in Fig 1 . The $\mathrm{Z}$ boson $v_{2}$
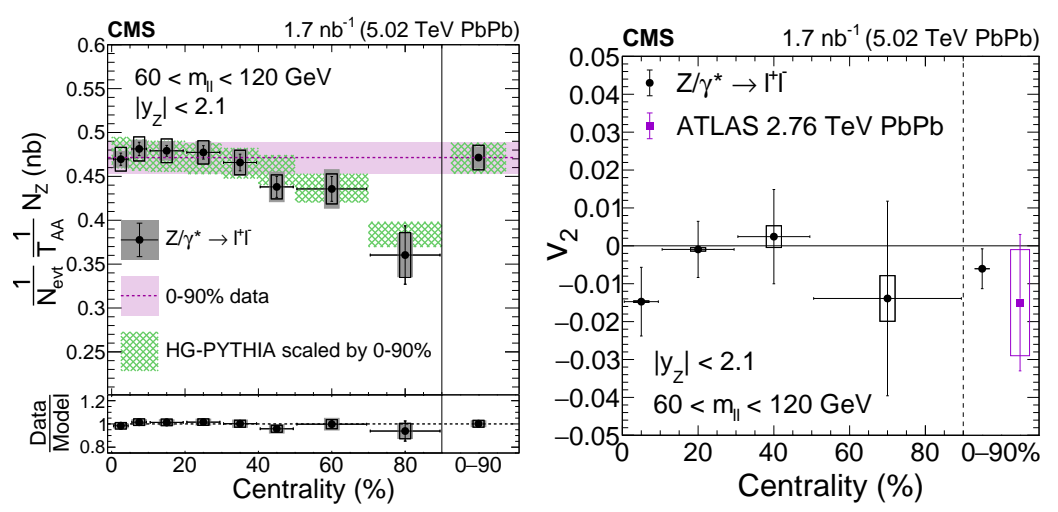

Figure 1: Left side figure shows the $T_{A A}$-normalized yields of $\mathrm{Z}$ bosons as a function of centrality [4]. The value of the $0 \%-90 \%$ data point and the scaled HG-PYTHIA model are shown for comparison, with the width of the bands representing the contribution from the total $0 \%-90 \%$ data point uncertainty. The right side figure shows the elliptic azimuthal anisotropy of $\mathrm{Z}$ boson in $\mathrm{PbPb}$ collision as a fuction of centrality [4].

measurement is compatible with zero within the statistical uncertainties [4] and significantly more precise than the previous ATLAS measurement [5]. It confirms that, since the $\mathrm{Z}$ boson is produced and decay very early in the collision and their leptonic daughters carry no color charge, it is largely unaffected by the final state effects such as hydro-dynamic flow and energy loss of the medium.

The $T_{A A}$ scaling can describe the measurements for central events (less than $40 \%$ ) as well as for the inclusive centrality $(0 \%-90 \%)$. However, a deviation is observed towards the peripheral collisions, indicating the presence of initial-state geometry and centrality calibration potential bias. Initialstate geometry effects can arise because bound nucleons have a spatial distribution that can shift the average nucleon-nucleon impact parameter away from that of proton-proton (pp) collisions [6]. 

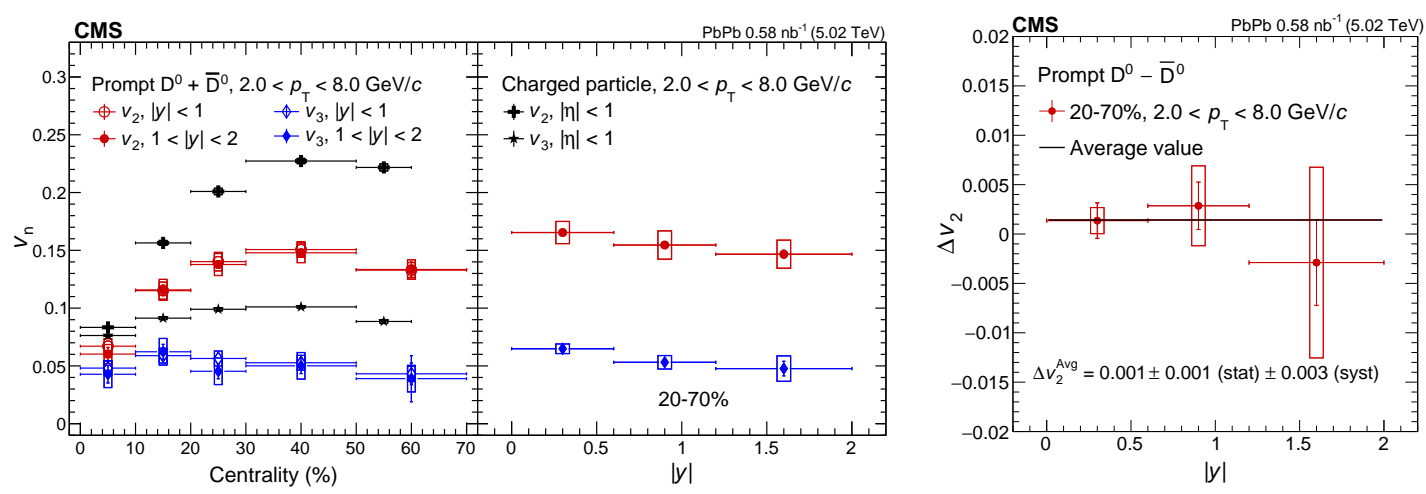

Figure 2: Left side figure shows the azimuthal anisotropies as a function of centrality and rapidity. Right side figure shows $\Delta V_{2}$ of prompt $D^{0}-\bar{D}^{0}[7]$.

The azimuthal anisotropies of prompt $D^{0}+\bar{D}^{0}$ in centrality and rapidity have been measured. Both in forward and mid-rapidity, the $v_{2}$ results show a clear increase in the value from central to mid-central region and declining towards peripheral collisions [7]. The right side plot of Fig 2. shows the rapidity average of $\Delta v_{2}$ in centrality $20 \%-70 \%$. For all rapidity bins, the values of $\Delta v_{2}$ is compatible with zero within the uncertainties. The average value in $|y|<2$ is $\Delta v_{2}=0.001 \pm 0.001$ (stat) \pm 0.003 (syst) [8]

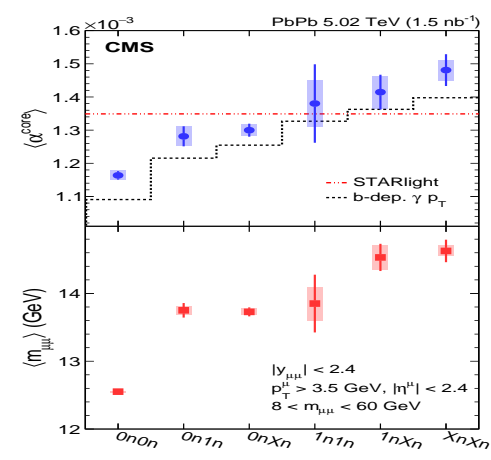

Figure 3: The above figure shows the neutron multiplicity dependence of the mean mass (bottom) and the core component of the average acoplanarity (top) of dimuons. [9]

A strong neutron multiplicity dependence of the core component of the average dimuon acoplanarity $\left\langle\alpha_{\text {core }}\right\rangle$ is measured [9]. Since the core part is formed from the leading order interaction, which is directly related to the photon $p_{T}$, the measurements show that the initial photon $p_{T}$ increases with the decrease of impact parameter. STARLIGHT MC [10] does not have this increasing trend but the CMS results are qualitatively described by the leading order QED model [11] calculation, which incorporates the impact parameter dependence of photon $p_{T}$. Our data results are five times higher than the model because of the absence of higher-order corrections for the soft photon radiation from the produced muon [12]. Also, the dimuon mean mass increases with increasing multiplicity since it is directly related to the initial photon energy. This also provides the information 
for the initial photon energy which is increasing with decreasing impact parameter [9].

\section{QGP medium effect}
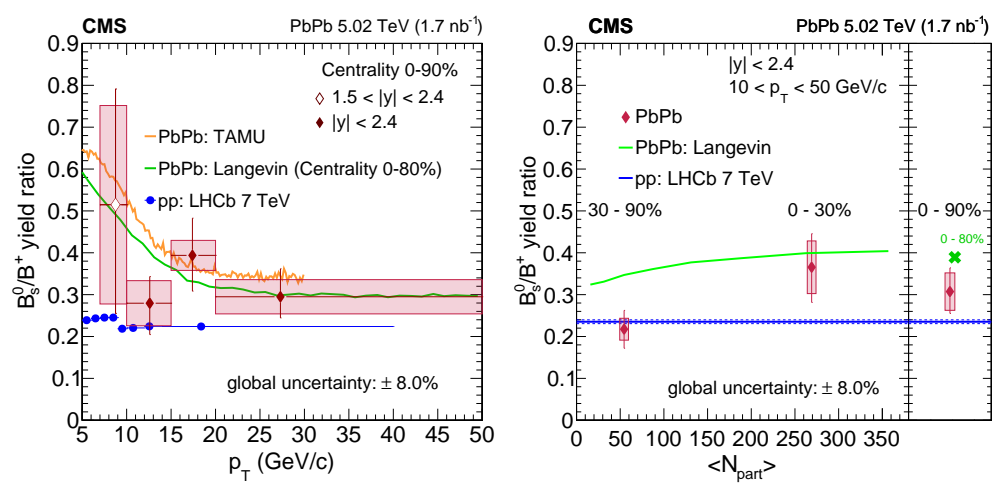

Figure 4: The ratio of $B_{s}^{0}$ and $B^{+}$meson production yields, as a function of $p_{T}$ (left) and collision centrality (right) [13].

The ratio of $B_{s}^{0}$ and $B^{+}$meson production has been studied for $|y|<2.4,1.5<y<2.4$ and $p_{T}$ $<50 \mathrm{GeV} / \mathrm{c}$ [13]. These results are compatible with the previously reported results by CMS [14] and based on a three-fold larger $\mathrm{PbPb}$ data sample. The ratio of production yields of the two mesons in $\mathrm{PbPb}$ collisions is determined and it is found to be statistically compatible with the corresponding ratio in pp results [15].
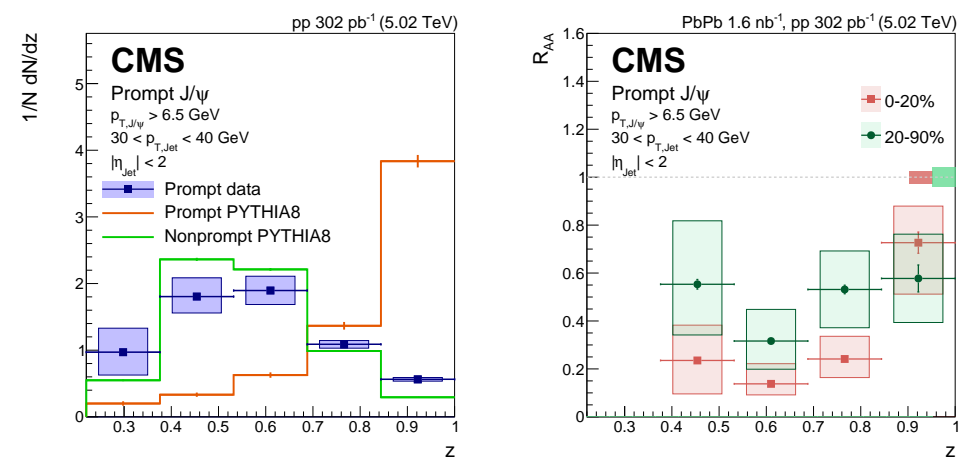

Figure 5: The prompt $J / \Psi$ normalized $\mathrm{z}$ distribution in pp collisions compared to PYTHIA8 calculations (left) [16] and nuclear modification factor $R_{A A}$ as a function of $\mathrm{z}$ for two centrality ranges of $\mathrm{PbPb}$ collisions (right) [16].

Measurements of the prompt $J / \Psi$ normalized z (the ratio of the $J / \Psi p_{T}$ to the $p_{T}$ of the jet into which it is clustered) distribution in pp data have been performed, as shown in Fig 5. Its shape is compared to PYTHIA 8 calculations for prompt and non-prompt $J / \Psi$. The $\mathrm{z}$ distribution in data is described by PYTHIA 8 for non prompt $J / \Psi$, which contains a larger jet-like component from fragmentation, as well as other products of the b-hadron decay. In the case of prompt $J / \Psi$, the 
data show a relatively large degree of surrounding jet activity, indicative of $J / \Psi$ production inside of parton showers. The value of $R_{A A}$ is estimated in two different centrality intervals $0 \%-20 \%$ and $20 \%-90 \%$. A large degree of suppression is observed for the more central collisions, which is expected because of the jet-medium interactions in QGP [16].

\section{Conclusion}

In this presentation we have discussed results from the initial and final state effects. Z-boson $v_{2}$ is compatible with zero within the statistical uncertainties while the yield spectra deviates from $T_{A A^{-}}$-scaling towards peripheral collisions, hinting the presence of initial-state geometry and centrality calibration potential bias. The average $D^{0}$ meson $\Delta v_{2}$ is measured and found to be $0.001 \pm 0.001$ (stat) \pm 0.003 (syst). This indicates there is no evidence that charm hadron collective flow is affected by strong Coulomb field generated in heavy-ion collisions. The dimuon mean mass and $\left\langle\alpha_{\text {core }}\right\rangle$ increases with the increase of neutron multiplicity. The $J / \Psi$ mesons formed within a jet are found to be more suppressed in central collisions than peripheral collisions. The ratio of production yields of the $B_{s}^{0}$ and $B^{+}$meson in $\mathrm{PbPb}$ collisions is determined and it is found to be statistically compatible with the corresponding ratio in pp results.

\section{References}

[1] Wit Busza et al., Annual Review of Nuclear and Particle Science 68, 339-376 (2018).

[2] Panagiota Foka et al., Reviews in Physics 1, 172-194 (2016).

[3] CMS Collaboration, JINST 03, S08004 (2008).

[4] CMS Collaboration, Phys. Rev. Lett. 127, 102002 (2021).

[5] ATLAS Collaboration, Phys. Rev. Lett. 802, 135262 (2020).

[6] Adam Bzdak et al., Phys. Rev. C 87, 064906 (2013).

[7] CMS Collaboration, Phys. Lett. B 816, 136253 (2021).

[8] Umut Gürsoy et al., Phys. Rev. C 98, 055201 (2018).

[9] CMS Collaboration, Phys. Rev. Lett. 127, 122001 (2021).

[10] Spencer Klein, arXiv:1607.03838.

[11] W Zha and Z Tang, J. High Energ. Phys. 83, 1029-8479 (2021).

[12] E J Williams, Phys. Rev. 45, 729 (1934).

[13] CMS Collaboration, Physics Letters B 796, 168-190 (2019).

[14] CMS Collaboration, Phys. Rev. Lett. 119, 152301 (2017).

[15] LHCb Collaboration, Phys. Rev. Lett. 124, 122002 (2020).

[16] CMS Collaboration, Physics Letters B 